\title{
$\mathbf{J}|\mathbf{A}| \mathbf{C} \mid \mathbf{S}$ \\ COMMUNICATIONS
}

Published on Web 04/11/2002

\section{A Simple Technique for Determining Nuclear Quadrupole Coupling Constants with RAPT Solid-State NMR Spectroscopy}

\author{
Subramanian Prasad, Hyung-Tae Kwak, Ted Clark, and Philip J. Grandinetti* \\ Department of Chemistry, Ohio State University, 120 West 18th Avenue, Columbus, Ohio 43210
}

Received February 12, 2002

Accurate measurements of the coupling between a nucleus' quadrupole moment and the local electric field gradient (efg), arising from surrounding electrons and nuclei, can provide atomic-level structural details on the coordination number and bonding geometry around a nucleus. ${ }^{1}$ The efg is described by a traceless second-rank tensor whose principal components are often expressed in terms of a quadrupole coupling constant $\left(C_{\mathrm{q}}\right)$ and an asymmetry parameter $\left(\eta_{\mathrm{q}}\right)$. The quadrupole moments of most quadrupolar nuclei are large enough that $C_{\mathrm{q}}$ values on the order of megahertz are found in all except the most symmetric bonding environments.

Several approaches exist for measuring $C_{\mathrm{q}}$ and $\eta_{\mathrm{q}}$ values with solid-state NMR. The simplest, which we classify as a secondorder method, is to perform a line shape analysis of the secondorder quadrupolar broadening of the central transition magic-angle spinning (MAS) spectrum. ${ }^{2}$ An advantage of this approach is that sensitivity is good since the second-order line width (in $\mathrm{Hz}$ ) is proportional to $C_{\mathrm{q}}{ }^{2} / v_{0}$, where $v_{0}$ is the Larmor frequency. A disadvantage is that the uncertainty in $C_{\mathrm{q}}$ and $\eta_{\mathrm{q}}$ increases with increasing $v_{0}$ or decreasing $C_{\mathrm{q}}$ as the features of the line shape become obscured by homogeneous line broadenings. A second approach, which we classify as a first-order method, is to perform a line shape (sideband) analysis of the first-order quadrupolar broadening of the satellite transition MAS spectrum. ${ }^{3}$ Here, the advantage is that the width is proportional to $C_{\mathrm{q}}$, reducing uncertainty of quadrupolar parameters in the presence of homogeneous line broadenings. At the same time, however, sensitivity is poorer, as the integrated intensity of the spectrum is spread over a wider frequency range, one that often exceeds the bandwidth of conventional NMR spectrometers. With both these approaches there is an additional disadvantage that overlapping anisotropic MAS line shapes from multiple sites can make line shape analysis intractable. Dynamic-Angle Spinning (DAS), ${ }^{4}$ Multiple Quantum Magic-Angle Spinning (MQ-MAS), ${ }^{5}$ and Satellite Transition Magic-Angle Spinning (ST-MAS) ${ }^{6}$ are second-order methods that address this issue with a two-dimensional spectrum correlating resolved isotropic resonances to separated second-order line shapes. The time needed to obtain sufficient sensitivity in these experiments can vary dramatically depending on the excitation efficiency of the required coherence transfer pathways. For MQ-MAS and ST-MAS excitation efficiencies are significantly reduced with increasing $C_{\mathrm{q}}{ }^{6,7}$ While DAS has comparatively better excitation efficiencies, mechanical difficulties have hindered its widespread adoption.

We have shown that Rotor Assisted Population Transfer (RAPT) ${ }^{8}$ preparation enhances the sensitivity of the central transition MAS spectrum for half-integer quadrupolar nuclei. In this communication, we describe an enhanced RAPT that also provides a simple and fast method to measure the magnitude of $C_{\mathrm{q}}$. With this approach, we obtain the bandwidth and sensitivity advantages of a second-

* Corresponding author. E-mail: grandinetti.1@osu.edu.

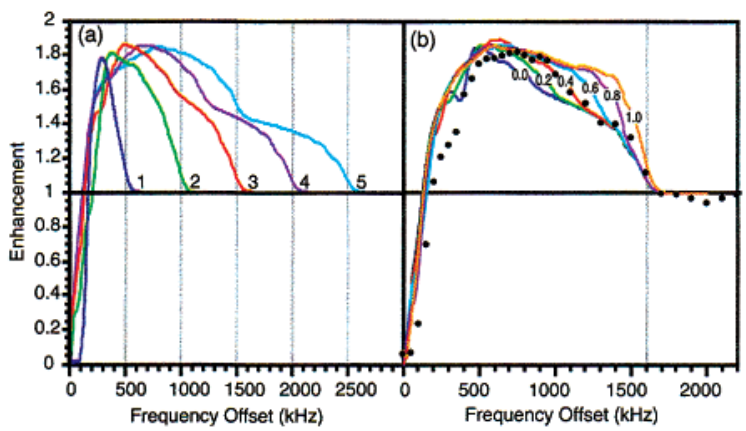

Figure 1. (a) Simulated Gaussian RAPT profiles for ${ }^{87} \mathrm{Rb}$ with $C_{\mathrm{q}}$ values as indicated and $\eta_{\mathrm{q}}=0.21$. (b) Simulated RAPT profiles for ${ }^{87} \mathrm{Rb}$ with $\eta_{\mathrm{q}}$ values as indicated and $C_{\mathrm{q}}=3.2 \mathrm{MHz}$. Solid dots in part b are the experimental ${ }^{87} \mathrm{Rb}$ RAPT enhancement profile for $\mathrm{RbClO}_{4}$. The RAPT preparation consisted of 20 Gaussian $\left(\sigma=2.855 \mu \mathrm{s}, \tau_{\mathrm{p}}=14.5 \mu \mathrm{s}\right)$ pulses alternating in offset frequency with a rf field strength of $72 \mathrm{kHz}$.

order method while retaining the $C_{\mathrm{q}}$ measurement precision of a first-order method. In addition, the RAPT sequence can be used to selectively suppress resonances based on their quadrupolar coupling constant. While this method does not provide an isotropic spectrum, it does provide a means of reducing the spectral complexity of multisite spectra.

The enhanced RAPT sequence consists of a train of Gaussian pulses with alternating off-resonant frequencies of $\pm v_{\text {off. }}$ This approach overcomes the need for fast phase shifting and allows us to explore a wider range of frequency offsets. The use of Gaussian pulses also improves the selectivity of satellite excitation. In Figure $1 \mathrm{a}$ is a plot of the RAPT sensitivity enhancement factor as a function of $\left|\nu_{\text {off }}\right|$ for a ${ }^{87} \mathrm{Rb}$ nucleus (spin 3/2) experiencing different $C_{\mathrm{q}}$ values. Enhancements increase to a maximum near $\left|v_{\text {off }}\right| \approx\left|C_{\mathrm{q}}\right| / 4$ and return to unity (no enhancement) when $\left|v_{\text {off }}\right| \geq\left|C_{\mathrm{q}}\right| / 2$. Figure $1 \mathrm{~b}$ illustrates the dependence of the RAPT sensitivity enhancement on $\eta_{\mathrm{q}}$ for a fixed $C_{\mathrm{q}}$ value of $3.2 \mathrm{MHz}$. With increasing $\eta_{\mathrm{q}}$ the RAPT enhancement profile becomes broader but more important, and it always returns to unity when $\left|v_{\text {off }}\right| \geq\left|C_{\mathrm{q}}\right| / 2$, independent of $\eta_{\mathrm{q}}$. This is a consequence of the outermost discontinuity (edge) of the first-order quadrupolar line shape for a satellite transition depending only on $C_{\mathrm{q}}$. Thus, measurement of the RAPT profile edge frequency, given by $v_{\text {edge }}=\left|3 C_{\mathrm{q}} /(2 I(2 I-1))\right|$, where $I$ is the spin of the nucleus, provides a simple means for determining $C_{\mathrm{q}}$. Additionally, the RAPT enhancement profile can provide information on $\eta_{\mathrm{q}}$, with steeper RAPT enhancement edges corresponding to higher $\eta_{\mathrm{q}}$ values.

The experimentally measured RAPT enhancement ${ }^{10}$ for ${ }^{87} \mathrm{Rb}$ in $\mathrm{RbClO}_{4}\left(C_{\mathrm{q}}=3.2 \mathrm{MHz}, \eta_{\mathrm{q}}=0.21\right)^{9}$ as a function of $\left|\nu_{\text {off }}\right|$ is shown as the solid circles in Figure 1b. We obtain excellent agreement between the observed $v_{\text {edge }}$ and the $C_{\mathrm{q}} / 2$ value of $1600 \mathrm{kHz}$. While the shape of the RAPT profile is also consistent with the lower $\eta_{\mathrm{q}}$ 


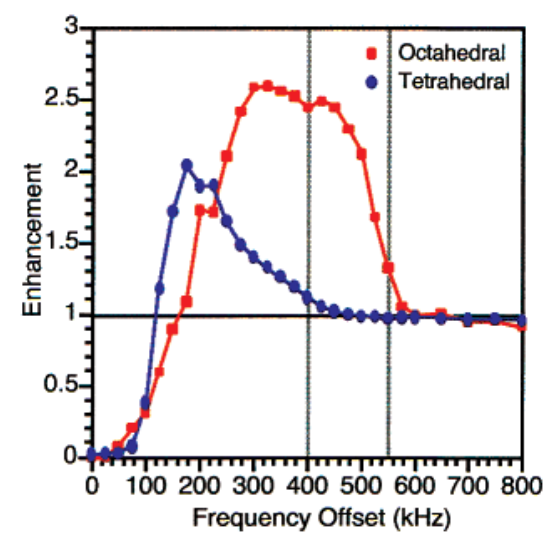

Figure 2. Experimental Gaussian RAPT profiles of ${ }^{27} \mathrm{Al}$ sites in VPI-5. The RAPT preparation consisted of 20 Gaussian $\left(\sigma=2.855 \mu \mathrm{s}, \tau_{\mathrm{p}}=16\right.$ $\mu \mathrm{s})$ pulses alternating in offset frequency with a rf field strength of $30 \mathrm{kHz}$.

value of $\mathrm{RbClO}_{4}$, obtaining $\eta_{\mathrm{q}}$ with this approach is not as reliable as measuring $C_{\mathrm{q}}$ from the RAPT profile edge frequency.

A possible source of error in measuring $C_{\mathrm{q}}$ with this approach arises from not being on-resonance with a site's isotropic chemical shift frequency when $v_{\text {off }}=0$, and from neglecting isotropic secondorder quadrupolar shifts. Another potential error source comes from neglecting higher order spinning sidebands of the satellite transition line shape. These sidebands will exist at frequencies beyond $\left|v_{\text {edge }}\right|$ and thus could obscure the RAPT enhancement edge position. We expect, however, that these errors will be on the order of a few kilohertz, and should lead to less than $1 \%$ error in $C_{\mathrm{q}}$ measurements. A more significant concern with large $C_{\mathrm{q}}$ values is that the observed RAPT enhancement edge could actually be an artifact of the NMR probe bandwidth. Thus, a slightly mismatched probe and higher rf power levels may be necessary when measuring larger $\left|v_{\text {edge }}\right|$ values.

To demonstrate this method in a higher spin system we examined the ${ }^{27} \mathrm{Al}$ (spin 5/2) resonances in VPI-5, a porous crystalline aluminophosphate molecular sieve. ${ }^{11}$ There are two tetrahedral sites in VPI-5 with overlapping resonances near $40 \mathrm{ppm}$ in the ${ }^{27} \mathrm{Al}$ MAS spectrum and an octahedral site with a well-defined second-order quadrupolar line shape at $-20 \mathrm{ppm}^{11}$ (spectrum not shown). The experimental RAPT enhancement as a function of $\left|v_{\text {off }}\right|$ is shown in Figure 2. For spin $5 / 2$ we use $C_{\mathrm{q}}=20\left|v_{\text {edge }}\right| / 3$, and obtain $C_{\mathrm{q}}=$ 3.67 MHz for the octahedral site, in good agreement with the earlier measured value of $3.5 \mathrm{MHz} .{ }^{11}$ Additionally, the RAPT enhancement edge is quite steep, also in agreement with the published $\eta_{\mathrm{q}}$ value of $0.91 .{ }^{11}$ For the tetrahedral sites it is more difficult to obtain a precise $C_{\mathrm{q}}$ value because of the overlap of sites. From the RAPT profile, we chose a $\left|v_{\text {edge }}\right|$ value of approximately $400 \mathrm{kHz}$ giving an estimated $C_{\mathrm{q}}$ of $2.67 \mathrm{MHz}$. The shape of the RAPT profile also suggests low $\eta_{\mathrm{q}}$ values. Unfortunately, $C_{\mathrm{q}}$ and $\eta_{\mathrm{q}}$ values are not known for these sites, and only the quadrupolar products $\left(P_{\mathrm{q}}=\right.$ $\left.C_{\mathrm{q}}\left(1+\eta_{\mathrm{q}}{ }^{2 / 2}\right)^{1 / 2}\right)$ of 1.3 and $2.8 \mathrm{MHz}$ have been reported. ${ }^{12}$ The RAPT measured value is in good agreement with the larger of these two values.

Finally, we note that RAPT can be employed for selective excitation or suppression of quadrupolar nuclei based on their quadrupolar coupling constant. For example, selective excitation of the resonance with the largest $C_{\mathrm{q}}$ value in a multisite spectrum can be obtained by presaturating the central transitions of all sites and then using RAPT to resurrect only the largest $C_{\mathrm{q}}$ resonance.

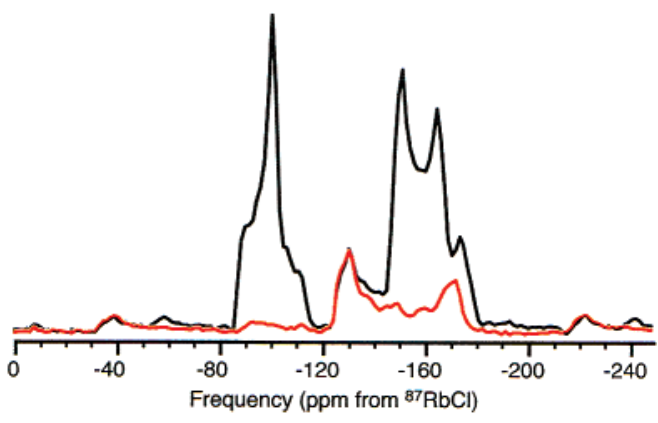

Figure 3. Shown in black is the conventional ${ }^{87} \mathrm{Rb}$ MAS spectrum of a $\mathrm{RbClO}_{4} / \mathrm{Rb}_{2} \mathrm{SO}_{4}$ mixture. In red is the MAS spectrum of the $C_{\mathrm{q}}=5.28$ $\mathrm{MHz}{ }^{87} \mathrm{Rb}$ resonance in $\mathrm{Rb}_{2} \mathrm{SO}_{4}$ selectively excited using the $\pi / 2$-selective RAPT sequence. Selective RAPT excitation was performed using $\left|v_{\text {off }}\right|=$ $1705 \mathrm{kHz}$ and consisted of 52 Gaussian $\left(\sigma=2.855 \mu \mathrm{s}, \tau_{\mathrm{p}}=13.5 \mu \mathrm{s}\right)$ pulses alternating in offset frequency with a rf field strength of $113 \mathrm{kHz}$.

We demonstrate this approach on the ${ }^{87} \mathrm{Rb}$ spectrum of a $\mathrm{RbClO}_{4} /$ $\mathrm{Rb}_{2} \mathrm{SO}_{4}$ mixture. In Figure 3, shown in black, is the conventional ${ }^{87} \mathrm{Rb}$ MAS spectrum of the mixture. $\mathrm{Rb}_{2} \mathrm{SO}_{4}$ contains two sites appearing at -100 and $-150 \mathrm{ppm}$ with $C_{\mathrm{q}}$ values of 2.67 and 5.28 $\mathrm{MHz}$, respectively. ${ }^{9}$ Overlapping with the $C_{\mathrm{q}}=5.28 \mathrm{MHz}$ site of $\mathrm{Rb}_{2} \mathrm{SO}_{4}$ is the $C_{\mathrm{q}}=3.2 \mathrm{MHz}$ site of $\mathrm{RbClO}_{4}$. By applying a soft $\pi / 2$ pulse prior to RAPT with $\left|v_{\text {off }}\right|=1705 \mathrm{kHz}$ we obtained the spectrum, shown in red, of only the $C_{\mathrm{q}}=5.28 \mathrm{MHz}$ site of $\mathrm{Rb}_{2} \mathrm{SO}_{4}$. Such a scheme can be employed to reduce spectral complexity in any NMR technique that draws its polarization from the central transition of half-integer quadrupolar nuclei. Extension of these ideas to higher spin nuclei is possible, and further work along these lines is in progress.

Acknowledgment. This material is based upon work supported in part by the National Science Foundation under Grant No. CHE 0111109, the U.S. Department of Energy, and the Ohio Supercomputer Center. Any opinions, findings, and conclusions or recommendations expressed in this material are those of the author(s) and do not necessarily reflect the views of the National Science Foundation (NSF).

\section{References}

(1) Lucken, E. A. C. Nuclear Quadrupole Coupling Constants; Academic Press Inc.: London, UK, 1969.

(2) Zheng, Z.; Gan, Z.; Sethi, N. K.; Alderman, D. W.; Grant, D. M. J. Magn. Reson. 1991, 95, 509.

(3) Skibsted, J.; Nielsen, N. C.; Bildsoe, H.; Jakobsen, H. J. J. Magn. Reson. 1991, 95, 88 .

(4) Chmelka, B. F.; Mueller, K. T.; Pines, A.; Stebbins, J.; Wu, Y.; Zwanziger, J. W. Nature 1989, 33942.

(5) Frydman, L.; Harwood, J. S. J. Am. Chem. Soc. 1995, 117, 5367.

(6) Gan, Z. J. Am. Chem. Soc. 2000, 122, 3242.

(7) Vosegaard, T.; Florian, P.; Massiot, D.; Grandinetti, P. J. J. Chem. Phys. 2001, 114, 4618

(8) Yao, Z.; Kwak, H.-T.; Sakellariou, D.; Emsley, L.; Grandinetti, P. J. Chem. Phys. Lett. 2000, 327, 85.

(9) Vosegaard, T.; Skibsted, J.; Bildsoe, H.; Jakobsen, H. J. J. Magn. Reson A $1996,122,111$.

(10) All NMR spectra were acquired on a Bruker DMX 400 spectrometer, using a $4 \mathrm{~mm}$ MAS probehead at spinning rates of $12 \mathrm{kHz} .{ }^{87} \mathrm{RbCl}$ and $1 \mathrm{M}^{27} \mathrm{Al}\left(\mathrm{NO}_{3}\right)_{3}$ solution were used to calibrate the rf field strength and as the external frequency reference. Gaussian pulses were defined in steps of $200 \mathrm{~ns}$.

(11) Rocha, J.; Kolodziejski, W.; He, H.; Klinowski, J. J. Am. Chem. Soc. $1992,114,4884$

(12) Rocha, J.; Esculcas, A. P.; Fernandez, C.; Amoureux, J.-P. J. Phys. Chem. 1996, 100, 17889.

JA025910Q 